\title{
Clinical Features of Pathological Processes of the Cervix Associated with Human Papillomavirus Infection
}

ISSN: 2577-2015

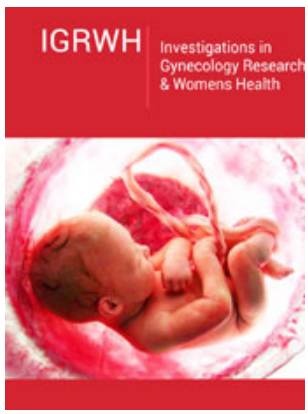

*Corresponding author: Unanyan A, Department of Obstetrics and Gynecology, Russia

Submission: 漈 October 25, 2019

Published: 侮 May 26, 2020

Volume 3 - Issue 4

How to cite this article: Unanyan $\mathrm{A}$ Pivazyan L, Kadirova A. Clinical Features of Pathological Processes of the Cervix Associated with Human Papillomavirus Infection. Invest Gynecol Res Women's Health. 3(4). IGRWH.000568. 2020. DOI: 10.31031/IGRWH.2020.03.000568

Copyright@ Unanyan A, This article is distributed under the terms of the Creative Commons Attribution 4.0 International License, which permits unrestricted use and redistribution provided that the original author and source are credited.

\author{
Unanyan A*, Pivazyan L and Kadirova A \\ Department of Obstetrics and Gynecology, Russia
}

\begin{abstract}
Background: Cervical Diseases associated with Papillomavirus infection are one of the most pressing problems modern gynecology due to the high frequency of occurrence (in the structure gynecological morbidity - from 20 to $45 \%$ ) and the ability to steady progression of the pathological process. A significant place in the structure of CM pathology is occupied by cervical intraepithelial neoplasia (CIN), which are often malignancy. Of particular importance are the issues of oncological transformation of the cervix in patients of reproductive age, taking into account the upcoming implementation of reproductive function. In Russia over the past 15 years, there has been an increase in the proportion of neglected cases of cervical cancer (cervical cancer), which ranks second (after breast cancer) in the structure of malignant tumors in reproductive age in women. Unfortunately, to date, the data of only an anamnesis, general clinical laboratory and instrumental methods of research are not productive enough to assess the risk of developing cervical onychopathology.
\end{abstract}

Objective: To identify the most significant clinical and anamnestic risk factors for the progression of pathological processes associated with human papillomavirus infection and the development of malignant transformation of the cervix in women of reproductive age, as they are important, taking into account statistical analysis.

Material and methods: The study conducted prospective and retrospective analyzes of 185 patients of reproductive age with cervical diseases; the age of the women surveyed ranged from 25-44 years.

Results: We have revealed a moderate relationship (using Spearman's correlation analysis) between the degree of progression of cervical disease and the presence of aggravated heredity (cancer of the reproductive system $)(\mathrm{R}=0.5679$, $\mathrm{p} 1.0$, which allowed us to attribute these factors of hereditary burden to cancer with statistically significant risk factors for malignant transformation and progression of the pathological process of cervix.

Conclusion: According to our study the most clinically and statistically significant risk factors are high virus HPV an oncogenic risk of more than 105 genomic equivalents; HPV persistence of high oncogenic risk; previously morphologically verified CIN; sexually transmitted mixed infections ( 3 or more).

Keywords: HPV; CIN; Cervical cancer; Gynecological oncology

\section{Introduction}

Cervical diseases associated with human papillomavirus (HPV) are an urgent problem of modern obstetrics and gynecology, primarily due to the high incidence and possibility of development of precancerous processes and cervical cancer [1]. Cervical intraepithelial neoplasia (CIN), which often leads to the development of cervical cancer, occupies a significant place in the structure of cervical diseases associated with HPV [2]. The current management and treatment tactics for detecting CIN are different: from observation using HPV testing and cytological control to excision, which in some cases is repeated, and even hysterectomy [1]. At the same time, the reason for such different tactics of management is not always unambiguous and needs a pathogenetic justification in order to identify the most effective approaches in the treatment of women of reproductive age. A factor requiring special attention in the management of women with CIN is the possibility of developing residual/recurrent disease [3]. Residual/recurrent disease is detected, as a rule, in patients with a neoplastic process at the edges of the resected tissue.

Currently, existing methods for predicting the progression of the residual neoplastic process, up to its malignant transformation, are not effective enough, and therefore the 
principles of the formation of high cancer risk groups require further development. Recently, an important role in the process of carcinogenesis has been played by epigenetic changes [4]. One of the main epigenetic events necessary for tumor development is acetylation of histones and methylation of tumor suppressor genes, which are the earliest events in the process of carcinogenesis [5]. However, the identification of their clinical significance and features in the genesis of the progression of pathological processes of CM require further research due to the insufficient number of works in this direction.

\section{Materials and Methods}

The study was conducted on the clinical bases of the Department of Obstetrics and Gynecology No. 1 (head of the department-MD, professor Ishchenko A.I.) First Moscow State Medical University named after I.M. Sechenov and LLC "Invitro". The study conducted prospective and retrospective analyzes of 185 patients of reproductive age with cervical diseases; the age of the women surveyed ranged from 25-44 years. In retrospect, all patients were divided into 5 groups depending on the morphological type of the pathological process of CM: group 1-patients with benign processes associated with HPV ( $n=32)$; Group 2-women with CIN I ( $n=43)$; Group 3-patients with CIN II (n=46); Group 4-women with CIN III $(n=42)$; Group 5-patients with invasive squamous cervical cancer (stage I; $n=22$ ). Patients with a morphologically verified diagnosis of CIN III or invasive squamous cervical cancer for further treatment were referred to an oncologist. Patients with CM adenocarcinoma were not found in the study.

Inclusion criteria-reproductive age, morphologically verified cases of pathological processes of cervix associated with HPV, HPVdetected HPV (high oncogenic risk), the presence of paraffin blocks in women with previous biopsy or excision of CM. Exclusion criteria: pregnancy and lactation, the use of HPV vaccine, severe somatic burden of patients (renal, hepatic, cardiovascular insufficiency), morphologically verified malignant formations (except squamous cervical cancer). In the process of determining urogenital infections, the diagnostic systems DNA-Sorb-AM, Amply Sense HPV Raman Screen-Titer-FL, Cobas 4800 (Roche Molecular Systems, USA) were used. Conducted traditional and liquid methods of cytological examination (classification of the Bethesda system), colposcopy (Sensitec OC-100 and SLC-2000). An aim biopsy and LLETZ, or conical excision, were performed on an EHVCh-140-02 Fotek device (Russia) and Surgitron DF 120 4.0 MHz (USA). An ultrasound scan was performed on an Acuson 128 XP 10 device (USA), Dornier AI 5200 “(Germany)," Logiq C5 “(USA). Destructive methods of treatment were performed using the PlasmajeT apparatus (Great Britain). The latest WHO classification [6] distinguishes between LSIL, whose synonym is CIN I, and HSIL, whose synonyms are CIN II and CIN III.

However, it should be noted that the classification that divides CIN into CIN I, CIN II, and CIN III [7] reflects in more detail the phased development of the neoplastic cervical process, which was the basis for the use of this particular classification in our own research work. All quantitative data obtained were processed by the method of variation statistics using spreadsheets "Microsoft Excel", as well as the application package "Statistica for Windows" v. 7.0, Stat Soft Inc. (USA). The probability of the occurrence of an event for some case was calculated by the formula, $p=1 /\left(1+e^{\wedge}(-z)\right.$ ) ,where $\mathrm{z}=\mathrm{b} 1 * \mathrm{X} 1+\mathrm{b} 2 \mathrm{xX} 2+\ldots+\mathrm{bnxXn+ \ldots X} \mathrm{X} 1$ are the values of independent variables, $\mathrm{b} 1$ are the coefficients, calculation which is the task of binary logistic regression, e is the natural logarithm and is equal to 3.714. In the resulting binary logistic regression equation, the prognosis (low risk-p<0.5) of the progression of the pathological process of cervix associated with HPV was calculated.

\section{Research Results and Discussion}

We carried out a detailed comparative analysis of clinical, medical and diagnostic parameters in the formed groups of patients. The average age of the examined patients was $38.4 \pm 1.4$ years. Using Pearson's correlation analysis, we found a direct moderate relationship between age and the degree of progression of the pathological process of cervix ( $\mathrm{r}=0.3485, \mathrm{p} 1.0$. It should be noted that, according to WHO 2013 (Guidelines for screening and treatment of precancerous lesions for cervical cancer prevention), the highest risk of cervical cancer occurs between the ages of 30 and 49. The age of women as a risk factor for the occurrence of pathological processes of cervical cancer has also been noted by other researchers [8].

We have revealed a moderate relationship (using Spearman's correlation analysis) between the degree of progression of cervical disease and the presence of aggravated heredity (cancer of the reproductive system) $(\mathrm{R}=0.5679, \mathrm{p} 1.0$, which allowed us to attribute these factors of hereditary burden to cancer with statistically significant risk factors for malignant transformation and progression of the pathological process of cervix. Hereditary factor, as a rule, is realized through the mechanism of predisposition to the disease, when and some inherited adaptive-protective mechanisms are not able to counteract exogenous carcinogenic effects [9]. Several researchers have noted a high degree of hereditary burden of patients in the development of tumor diseases of the genital organs, which, in their opinion, is an important factor predisposing to the development of cervical disease [10].

When studying somatic diseases, it was found that the differences in the frequency of diseases of the respiratory, urinary, nervous systems, gastrointestinal tract in the comparison groups were statistically insignificant $(p>0.05)$. In the study of reproductive history, it is noteworthy that the number of births and abortions did not statistically significantly differ between the groups, however, a significant predominance of post-abortion and postpartum traumatic cervical injuries was revealed in women with CIN III (83.3\%) and invasive cervical cancer $(90,9 \%)$ compared with patients in the first three groups $(9.4 \%, 11.6 \%$ and $43.5 \%$, respectively); $(\mathrm{p}<0.05)$. Traumatic injuries of cervix (postpartum, post-abortion, etc.) in the progression of the pathological process of cervix were identified as a significant risk factor based on the calculation of RR. According to Prilepskaya et al. [10] traumatic injuries of cervix with its further deformation lead to a violation of the physiological barrier, which is accompanied by a decrease 
in local immunity and easier penetration of the infection. Data from other researchers indicate that precancer and cervical cancer are often formed against the background of post abortion and postpartum cicatricial deformity of cervical cancer and ectropion [11].

Using Pearson's correlation analysis, we revealed a direct moderate dependence between the duration of smoking and the degree of progression of the pathological process of cervix $(r=0.4176, p<0.0001)$, which indicates a tendency to increase the incidence of cervical diseases with long-term smoking. Tobacco smoking for more than 7 years was chosen as a factor in the progression of the neoplastic cervical process in connection with the highest possible RR compared to other similar indicators, and also taking into account the fact that the lower limit of $95 \% \mathrm{CI}$ is higher than 1, which indicates the statistical significance of this factor. According to numerous studies, smoking women compared with non-smokers are 2-3 times higher risk of developing cervical cancer [12].

Noteworthy data on infections in the examined patients. The most significant increase in the frequency of mixed infections (combination of 3 or more) in the comparison groups from 1 (3.1\%) to $5(72.7 \%)$ in the examined patients was revealed; ( $<<0.05)$, as well as recurrent bacterial vaginosis in the same comparison groups in $1(9.4 \%)$ and 5 (86.4\%); ( $<<0.05)$. Mixed infection factors (a combination of 3 or more) and recurrent bacterial vaginosis due to the highest RR and a CI above 1 were evaluated as statistically significant. The findings of other studies indicate that the presence of mixed infections can contribute to the development of dystrophic changes in stratified squamous epithelium, disruption of intercellular contacts, leukocyte and lymphocytic infiltration of the stroma [13]. A systematic review by Murta et al. [14] confirms the significant association between bacterial vaginosis and CIN [14].

In our own study, a high frequency of chronic cervicitis was found in women with CIN II (58.7\%), CIN III (59.5\%) and squamous cervical cancer $(86,4 \%)$; $(\mathrm{p}<0.05)$ relative to this condition in the comparison groups, which, along with the indicators of RR and CI, made it possible to identify chronic cervicitis as a risk factor for the progression of cervical pathology. According to Lebedeva et al. [15], in $47.1 \%$ of patients with chronic cervicitis there is a high frequency of infection with HPV-BP. According to several other authors, chronic cervicitis is one of the main exogenous factors contributing to the development of dysplasia and cervical cancer [16].

When analyzing the characteristics of sexual life and contraception in the examined women, statistically significant indicators of the early onset of sexual activity (up to 18 years of age, without the use of barrier contraception) and the number of sexual partners more than 7 (from the moment of the onset of sexual activity) in women $4(38,1 \%)$ and $5(36.4 \%)$ groups compared with similar indicators in patients of groups 1,2 and 3 (9.4\%, $16.3 \%$ and $21.7 \%$, respectively); $(\mathrm{p}<0.05)$. The calculation of the RR factor of the number of sexual partners of more than 7 (since the onset of sexual activity) and the onset of sexual activity up to 18 years (without the use of barrier contraception) made it possible to single out this factor as a statistically significant criterion in the progression of cervical pathology. The relationship between HPV infection and promiscuous sexual life has been noted in other studies [1].

The analysis of the clinical picture in patients with pathological processes of cervix shows that the clinical symptoms in patients with cervical diseases are usually due to the presence of a combined uterine pathology (endo- and myometrium). The combination of uterine fibroids with adenomyosis and/or endometrial hyperplastic process in patients with CIN III (47.6\%) and cervical cancer (59.1\%) was much more frequent compared with the incidence of combined uterine pathology in women of comparison groups; $(\mathrm{p}<0.05)$. This fact, apparently, is due to the mutually stimulating effect of pathological processes of endo- and myometrium on the state of cervix, which is realized through the mechanisms of intercellular interactions of growth factors. According to Sidorova et al. [16], there are significant differences in the incidence of CIN in women with combined uterine pathology compared with isolated uterus pathology $(\mathrm{p}<0.001)$, where the relative risk of CIN in women with combined uterine diseases (uterine fibroids, adenomyosis, endometrial hyperplasia) is 62 times higher than women with any isolated uterine disease [16].

Our study revealed the persistence of HPV-BP in 80 women (43.2\%), the viral load of HPV-BP more than 105 genomic equivalents in 83 women (44.9\%), as well as the simultaneous combination of two or more types of HPV-VR infection in 96 women (51.9\%). An analysis of HPV infection and the calculation of RR allowed us to determine the persistence of HPVR, the viral load of HPV-HR more than 105 genomic equivalents, and the simultaneous combination of two or more types of HPV-HR as significant factors in the progression of the neoplastic process. Literature data indicate that one-year or two-year persistence, especially HPV type 16, is a high-risk factor for the development of CIN 3 or 3+ [1]. In the study Rogovskaya et al. [1] combined HPV infection is more common in patients with HSIL (58\%) than in patients with latent papillomavirus infection (PVI) and LSIL (46\%) [1]. Data from other literature sources also indicate that viral load is associated with a degree of CIN but indicating that viral load as the only marker for determining the risk of neoplastic transformation is not sufficiently informative $[1,16]$.

An assessment of the cases of a morphologically verified CIN in the anamnesis (this fact was recorded when requesting paraffin blocks and reviewing the "glasses" of the preparation) revealed its statistical significance with respect to the possible progression of the pathological process of CM in 4 (28.6\%) and 5 (31.8\%) groups compared with the other three groups $(3.1 \%, 9.3 \%$ and $15.2 \%$, respectively); $(\mathrm{p}<0.05)$.

Thus, the results of clinical and medical history and diagnostic features in the studied groups of patients allowed us to determine statistically significant risk factors for the development of malignant transformation of CM. To further, improve the approach to the formation of high cancer risk groups. We carried out the next 
stage of the study, aimed at identifying pathogenic risk factors for the development of cervical cancer, by a detailed analysis of the molecular biological characteristics of acetylation and methylation with the above clinical risk factors in pathological processes of cervical cancer associated with HPV.

\section{Conclusion}

The most clinically and statistically significant risk factors (in terms of significance) of the progression of the pathological process associated with HPV and the risk of developing malignant transformation of the cervix uteri are (with the highest possible $\mathrm{RR}>1$ and with a lower limit of $95 \% \mathrm{CI}>1$ ): high virus HPV an oncogenic risk of more than 105 genomic equivalents; HPV persistence of high oncogenic risk; previously morphologically verified CIN; sexually transmitted mixed infections (3 or more); recurrent bacterial vaginosis; traumatic injuries of the cervix (postpartum, post abortion); age more than 40 years; early onset of regular sexual activity (up to 18 years) without the use of barrier methods of contraception; more than 7 sexual partners (since the sexual debut); the simultaneous combination of two or more types of HPV high oncogenic risk; chronic cervicitis; hereditary burden of cancer of the organs of the reproductive system; hereditary burden of common oncological diseases; a combination of uterine fibroids with adenomyosis and/or endometrial hyperplastic process; smoking.

\section{References}

1. Rogovskaya SI, Lipova EV (2014) Cervix vagina, vulva. A Guide for Doctors, p. 830.

2. Korolenkova LI (2014) Clinical and morphological conception of the occurrence and course of severe CIN. Clinical Laboratory Diagnostics 6: 18-22.

3. Ulrich D, Tamussino K, Petru E, Haas J, Reich O (2012) Conization of the uterine cervix: does the level of gynecologist's training predict margin status? Int J Gynecol Pathol 31(4): 382-386.

4. BarrowTM, Michels KB (2014) Epigenetic epidemiology of cancer. Biochem Biophys Res Commun 455(1-2): 70-83.

5. Kiselev VI, Drukh VM, Kuznetsov IN, Muizhnek EL, Apolikhina IA, et al. (2014) Evaluation of the effectiveness of drug therapy of cervical intraepithelial neoplasia of mild to moderate severity with CerviconDIM suppositories (interim results of a clinical trial). Gynecology 6: 5862.

6. WHO (2014) Classification of tumors of female reproductive organs is the sixth volume in the Fourth Edition of the WHO series on histological and genetic typing of human tumors? In: Robert JK, Maria LC, Herrington s, et al. (Eds.), Young ( $4^{\text {th }}$ edn), Switzerland, p. 316.

7. WHO (2003) Pathology and genetics of tumors of the breast and female genital organs (IARC who classification of tumors)? Lyon, France.

8. Saslow D, Solomon D, Lawson HW, Killackey M, Kulasingam SL, et al. (2012) American cancer society, American society for colposcopy and cervical pathology, and American society for clinical pathology screening guidelines for the prevention and early detection of cervical cancer. Am J Clin Pathol 137(4): 516-542.

9. Nazarova NM, Prilepskaya VN, Sulamanidze LA, Mzarelua GM, Bestaeva NV (2013) Papillomavirus infection: prevalence, diagnosis and treatment. Attending Physician 11: 15-18.

10. Prilepskaya VN, Bebneva TN (2011) Primary prevention of cervical cancer: Achievements and prospects. Farmateka 13: 42-46.

11. Radzinsky VE (2013) Problems of diagnosis and treatment of diseases of the cervix, vagina and vulva VE Radzinsky. Post-release and materials of the scientific program of the International Interdisciplinary Forum "Cervix and Vulvovaginal Diseases". M: Editorial Office of the Journal Status Praises, p. 3-4.

12. Mirabello L, Schiffman M, Ghosh A, Rodriguez AC, Vasiljevic N, et al. (2013) Elevated methylation of HPV16 DNA is associated with the development of high grade cervical intraepithelial neoplasia. Int J Cancer 132(6): 1412-1422.

13. Strizhakov AN, Belotserkovtseva LD, Budanov PV (2014) A systematic approach to the selection of a clinical solution for vulvovaginal infections. Gynecology, Obstetrics and Perinatology 1: 60-66.

14. Murta EF, Souza MA, Araújo E, Adad SJ (2000) Incidence of Gardnerella vaginalis, Candida SP and human papilloma virus in cytological smears. Sao Paulo Med J 118(4): 105-108.

15. Lebedeva LM (2009) Papillomavirus infection in inflammatory diseases of the cervix. Pathogenetic Approaches to Treatment, p. 22.

16. Sidorova IS, Unanyan AL, Zaletaev AV, Sha S, Zholobova MN, et al. (2012) The role of microsatellite instability of the genome in diseases of the cervix uteri in women with uterine myoma, adenomyosis, endometrial hyperplasia and their combination. Russian Journal of Obstetrician Gynecologist 4: 13-16. 